\title{
INCREMENTAL DISPLACEMENT ESTIMATION METHOD FOR VISUALLY SERVOED PARIED STRUCTURED LIGHT SYSTEM (VISP)
}

\author{
H. Jeon ${ }^{1}$, J. U. Shin ${ }^{2}$, W. Myeong ${ }^{1}$, and H. Myung ${ }^{1,2}$ \\ ${ }^{1}$ Department of Civil and Environmental Engineering, KAIST (hmyung@kaist.ac.kr) \\ ${ }^{2}$ Robotics Program, KAIST
}

\begin{abstract}
With the continuing requirement of the structural displacement monitoring for massive civil structures, a vision-based displacement monitoring system is gaining great attention as an inexpensive solution. Currently, a visually servoed paired structured light system (ViSP) which measures the relative translational and rotational displacement each in $3 D O F$ has been researched and the various simulation and experiments verified the performance of the system. The system is composed of two sides facing with each other, each with one or two lasers, a 2-DOF manipulator, a camera, and a screen. The 6-DOF displacement is calculated from the positions of the projected laser beams and the rotation angles of the manipulators on both sides by using iterative method such as extended Kalman filter (EKF) or Newton-Raphson. Since the computation time is relatively long due to the multiple iterations, this paper proposes non-iterative novel incremental displacement estimation (IDE) method that updates the displacement by using the previously estimated displacement and the difference of the previous and the current observed data. To verify the performance of the proposed method, a simulation has been performed and the result shows that the proposed non-iterative method estimates the displacement with the same level of accuracy in significantly reduced computation time compared to the EKF with multiple iterations.
\end{abstract}

Keywords: Structural Health Monitoring (SHM), displacement, Visually Servoed Paired structured light system (ViSP), Incremental Displacement Estimation (IDE)

\section{INTRODUCTION}

Since civil structures are exposed to various external loads such as traffic, earthquake, and wind load, it is essential to evaluate the structural safety in any moment. For this reason, structural health monitoring (SHM) has been widely studied for several decades [1]. To estimate structural displacement, one of the important categories of SHM, accelerometers, global positioning systems (GPS), and laser Doppler vibrometers (LDVs) have been used. However, these sensors have problems that the accelerometers indirectly estimate the displacement by integrating the acceleration twice, the cost of GPS with high resolution is significantly high, and LDVs need reference position for installation and the cost is also high. 
To mitigate the aforementioned problems, a vision-based displacement monitoring system has been researched [2, 3]. Most of the vision-based monitoring system installs the targets on the structure and the camera from a far captures the images of the targets. Since the distance between the camera and the target is relatively long, it is highly sensitive to the external environmental changes such as weather or illumination. In other words, the displacement can be calculated only when the line of sight is guaranteed. Also it estimates only 2DOF translational displacement.

Therefore, Myung et al. proposed a paired structured light system composed of two sides facing with each other, each with one or two lasers, a camera, and a screen [4]. The lasers on each sides projects their parallel beams to the screen on the opposite side and the camera near the screen captures the image of the screen. Due to this short distance, the system is robust to the external changes. Also, the translational and rotational displacement each in 3DOF can be estimatedwjs132 by calculating the positions of the totally three projected laser beams. Though the performance of the system has been verified from the previous study [4], it has a major problem that the measurable range is limited due to the limited screen size. To solve the problem, a visually servoed paired structured light system (ViSP) was newly proposed by Jeon et al. [5]. In this system, 2-DOF manipulator is added to control the pose of the lasers for the projected lasers beams to be on the screen all the times.

In the displacement estimation process, an iterative method such as extended Kalman filter (EKF) or Newton-Raphson have been used. However, the computation time of these methods is relatively long due to the multiple iterations. Therefore, in this paper, incremental displacement estimation (IDE) method which updates previously estimated displacement with the difference of the previous and the current positions of the projected laser beams. In Section 2, the architecture and the kinematics of the ViSP are described. In Section 3, the incremental displacement estimation method is proposed. In Section 4, a simulation is introduced to validate the performance of the proposed system. Concluding remarks are discussed in Section 5.

\section{VISUALLY SERVOED PAIRED STRUCTURED LIGHT SYSTEM (ViSP)}

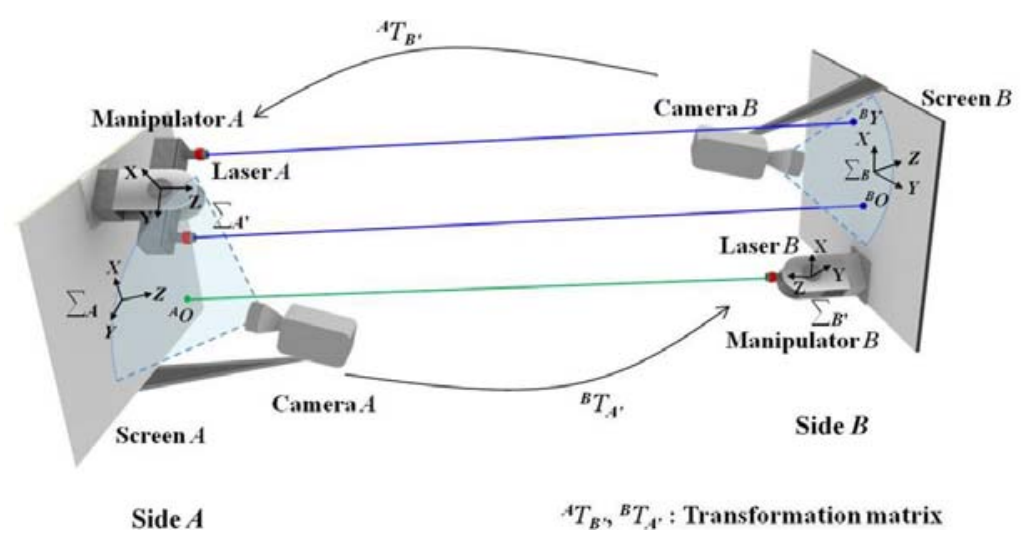

Figure 1. Schematic diagram of the visually servoed paired structured light system (ViSP) [5] 
The schematic diagram of the visually servoed paired structured light system (ViSP) is shown in Figure 1. As shown in the figure, each side is composed of one or two lasers with a 2-DOF manipulator, a camera, and a screen. Each laser projects its parallel beam to the screen on the opposite side and the camera near the screen captures the image of the screen. Since the distance between the camera and the screen is short, less than $20 \mathrm{~cm}$, the system is robust to the environmental changes. The manipulator on each side controls the pose of the lasers for the projected laser beams to be on the screen before it gets off the screen boundary. The 6DOF relative displacement between two sides can be calculated from positions of the three projected laser beams and the rotation angles of the manipulators.

The kinematic equation of the system defines the geometric relationship between the observed data $m=\left[{ }^{A} O,{ }^{B} O,{ }^{B} Y\right]^{\mathrm{T}}$ and the estimated displacement $\left.p=[x, y, z, \theta, \varphi, \psi]\right]^{\mathrm{T}}$. Here ${ }^{A} O$ is the projected laser beam on the screen $A$, and ${ }^{B} O$ and ${ }^{B} Y$ are the projected laser beams on the screen $B . x, y$, and $z$ are the translational displacement along $X, Y$, and $Z$ axis, respectively and $\theta, \varphi$, and $\psi$ are the rotational displacement about $X, Y$, and $Z$ axis, respectively. To derive the kinematics, the transformation matrices ${ }^{A} T_{B}$ and ${ }^{B} T_{A}$ can be used. The ${ }^{A} T_{B}$ is a transformation matrix that transforms the coordinate from screen $B$ to screen $A$. Using the transformation matrix ${ }^{A} T_{B}$ and the position of the laser installed, the ${ }^{A} O$ can be obtained as follows:

$$
{ }^{A} O={ }^{A} T_{B} \cdot{ }^{B} T_{B^{\prime}}\left[\begin{array}{llll}
0 & 0 & Z_{A B} & 1
\end{array}\right]^{T} .
$$

In Eq. (1), $Z_{A B}$ is the distance from screen $A$ to screen $B,{ }^{B} T_{B^{\prime}}$ is the rotation matrix consists of encoder information of the manipulator on side $B,{ }^{B} T_{B^{\prime}}=R_{x}\left(-\theta_{\text {Benc }}\right) \cdot R_{z}\left(-\psi_{\text {Benc }}\right)$ where $\theta_{\text {Benc }}$ and $\psi_{B e n c}$ are rotated angles of the manipulator $B$ about $X$ and $Z$ axis, respectively. In the same way, ${ }^{B} O$ and ${ }^{B} Y$ can be easily obtained as follows:

$$
\begin{gathered}
{ }^{B} O={ }^{B} T_{A}{ }^{A} T_{A^{\prime}}\left[\begin{array}{llll}
0 & -L & Z_{A B} & 1
\end{array}\right]^{T}, \\
{ }^{B} Y={ }^{B} T_{A} \cdot{ }^{A} T_{A^{\prime}}\left[\begin{array}{llll}
0 & L & Z_{A B} & 1
\end{array}\right]^{T}
\end{gathered}
$$

where $L$ is the offset of the laser point from the center of a screen in $Y$ direction, ${ }^{A} T_{A}$, is the rotation matrix consists of encoder information of the manipulator on side $A$, and ${ }^{A} T_{A^{\prime}}=R_{x}(-$ $\left.\theta_{\text {Aenc }}\right) \cdot R_{z}\left(-\psi_{\text {Aenc }}\right)$ where $\theta_{\text {Aenc }}$ and $\psi_{\text {Aenc }}$ are rotated angles of the manipulator $A$ about $X$ and $Z$ axis, respectively. As $z=0$ on screens, $z$ component of ${ }^{A} O,{ }^{B} O$, and ${ }^{B} Y$ vectors should be zero. By applying these constraints, the kinematic equation can be derived as follows:

$$
M_{v s}=\left[\begin{array}{llllll}
{ }^{A} O_{x} & { }^{A} O_{y} & { }^{B} O_{x} & { }^{B} O_{y} & { }^{B} Y_{x} & { }^{B} Y_{y}
\end{array}\right]^{T}
$$

where ${ }^{A} O_{x}$ and ${ }^{A} O_{y}$ denote the $x$ and $y$ component of ${ }^{A} O$, respectively.

By using the iterative method such as the EKF or the Newton-Raphson method, the estimation of $p$ can be obtained. In this paper, however, the newly proposed incremental displacement estimation (IDE) method is used considering a measurement noise which occurs when detecting the center of the laser beams and the computation time. 


\section{INCREMENTAL DISPLACEMENT ESTIMATION (IDE)}

Although the iterative method such as the EKF or Newton-Raphson can estimate displacement with high accuracy, the computation time is relatively long due to the multiple iterations. To reduce the computation time, the non-iterative incremental displacement estimation (IDE) method which is a modified version of the EKF is proposed [6]. In this method, the previously estimated displacement $(\hat{p}(t \mid t))$ and the difference of the previous and the current observed data $m(\Delta m)$ is used for the prediction step. The prediction of the displacement $(\hat{p})$, measurement $(\hat{m})$, and displacement $(P)$ can be obtained as follows:

$$
\begin{aligned}
& \hat{p}(t+1 \mid t)=\hat{p}(t \mid t)+J_{p}^{+}(t) \cdot \Delta m(t+1 \mid t) \\
& \hat{m}(t+1 \mid t)=M_{v s}(\hat{p}(t+1 \mid t)) \\
& P(t+1 \mid t)=P(t)+Q
\end{aligned}
$$

where $\Delta m(t+1 \mid t)$ is the difference of the previous and the current positions of the projected laser beams calculated by $\Delta m(t+1 \mid t)=m(t+1)-\tilde{m}(t)$ where $\tilde{m}(t)$ are the positions of the projected laser beams compensated by the current rotation angles of the manipulator. $J_{p}^{+}(t)$ is the pseudo-inverse of Jacobian of Eq. (4) and $(t+1 \mid t)$ means a priori estimate.

In the observation step, the followings equation is applied:

$$
\begin{aligned}
& v(t+1)=m(t+1)-\hat{m}(t+1 \mid t) \\
& S(t+1)=J_{P}(t+1) \cdot P(t+1 \mid t) \cdot J_{P}{ }^{T}(t+1 \mid t)+R .
\end{aligned}
$$

where $R$ is the covariance matrix of the measurement noise.

The update step can be applied to obtain a posteriori result as follows:

$$
\begin{aligned}
& K(t+1)=P(t+1 \mid t) J_{p}^{T}(t+1) S^{-1}(t+1) \\
& \hat{p}(t+1 \mid t+1)=\hat{p}(t+1 \mid t)+K(t+1) v(t+1) \\
& P(t+1 \mid t+1)=P(t+1)-K(t+1) S(t+1) K^{T}(t+1) .
\end{aligned}
$$

where $P$ is the displacement error covariance matrix, $K$ is the Kalman gain, and $(t+1 \mid t+1)$ means a posteriori estimate.

\section{SIMULATION}

To validate the performance of the proposed IDE method, a simulation of estimating the dynamic displacement was conducted. In this simulation, the displacement data, $g(t)$, are obtained from the benchmark study proposed by Spencer et al. [7]. The dynamic displacement is estimated using ViSP with three different method; IDE, EKF with 50 iterations $(\operatorname{EKF}(50))$, and $\mathrm{EKF}$ with 1 iteration $(\operatorname{EKF}(1))$. The displacement results with each different method were evaluated against the root mean square error (RMSE) and the coefficient of the cross-correlation (CORR). The displacement results are shown in Table 1 and Figure 2. As shown in the results, the proposed non-iterative IDE method significantly reduced the computation time by $98 \%$ with the same level of accuracy compared to $\operatorname{EKF}(50)$. 
Table 1 Root mean square errors (RMSE) and cross-correlation coefficients (CORR) of dynamic displacement estimation using three methods; the proposed incremental displacement estimation (IDE) method, the EKF with 50 iterations $(\mathrm{EKF}(50))$, and the EKF with 1 iteration $(\operatorname{EKF}(1))$, respectively [6].

\begin{tabular}{lcccc}
\hline Method & $\begin{array}{c}\text { RMSE } \\
(\mathrm{mm})\end{array}$ & $\begin{array}{c}\text { RMSE } \\
\left({ }^{\circ}\right)\end{array}$ & CORR & $\begin{array}{c}\text { Computation time } \\
(\mathrm{msec} / \text { cycle })\end{array}$ \\
\hline \hline IDE & 0.34 & 0.02 & 0.99 & 0.10 \\
$\operatorname{EKF}(50)$ & 0.56 & 0.15 & 0.99 & 4.25 \\
$\operatorname{EKF}(1)$ & 1.20 & 0.05 & 0.98 & 0.09 \\
\hline
\end{tabular}
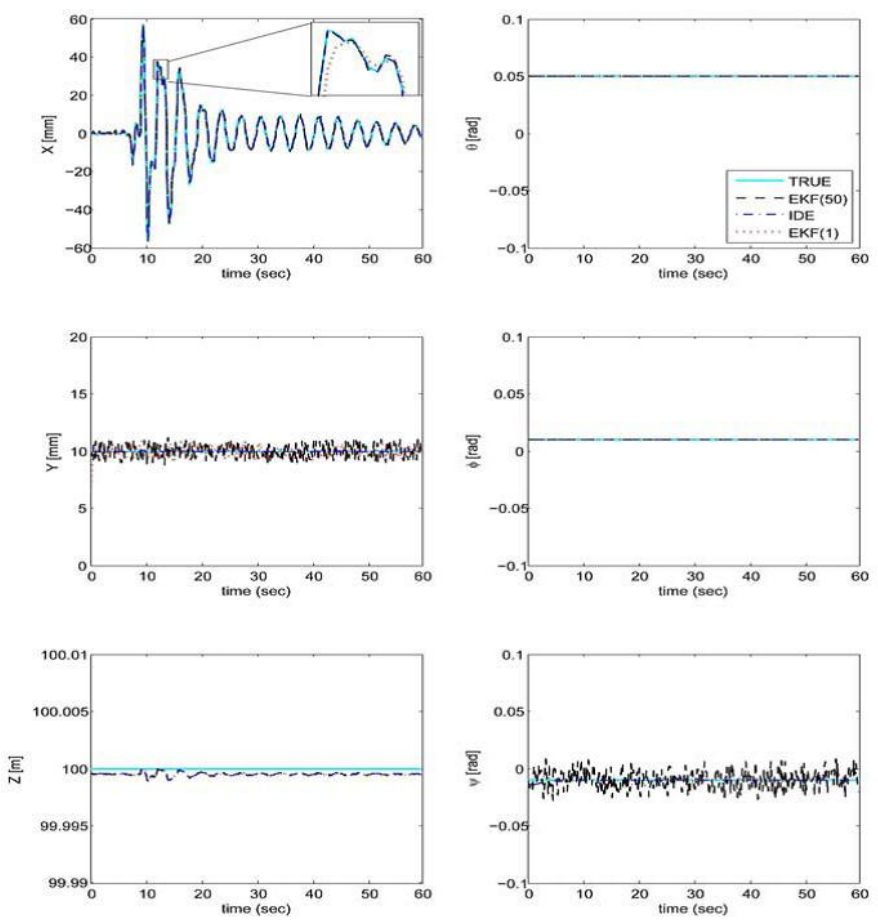

(a)

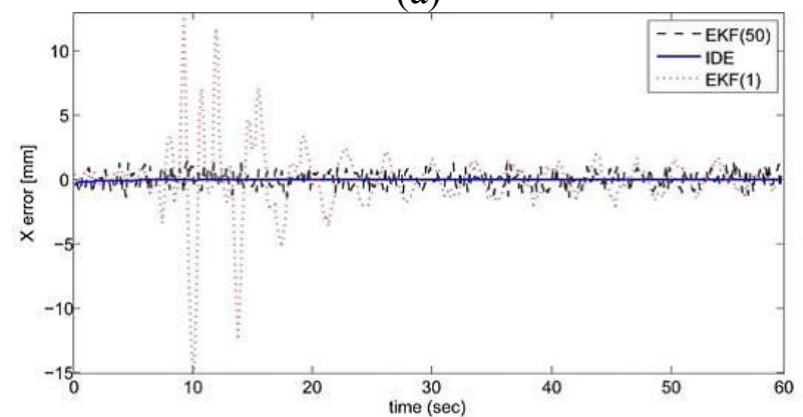

(b)

Figure 2 Simulation results. (a) The estimated 6-DOF displacement from three methods. Dashed line: estimated displacement by using the EKF with the 50 iterations $(\operatorname{EKF}(50))$. Dashed dot line: estimated displacement by using the proposed incremental displacement estimation (IDE) method. Dotted line: estimated displacement by using the EKF with 1 iteration $(\operatorname{EKF}(1))$. Solid line: the true value of $\left(x_{t}, \underline{y}_{t}, z_{t}, \theta_{t}, \Phi_{t}, \psi_{t}\right)=(g(t), 0.01,100,0.05,0.01$, -0.01), where all units are in meters and radians. (b) The error of the estimated $X$-axis translational displacement from each method. Dashed line: error of the EKF(50). Solid line: error of the IDE. Dotted line: error of the EKF(1) [6]. 


\section{CONCLUSIONS}

In this paper, an incremental displacement method for 6-DOF displacement estimation was proposed. Similar to EKF, the method is composed of prediction, observation, and update steps. In this methodthe displacement is estimated from the previously estimated displacement with the difference between the previous and the current positions of the projected laser beams. To validate the performance of the proposed method, a simulation with the dynamic displacement data was performed. The result shows that the proposed method estimates the displacement with a same level of accuracy in comparison with EKF with 50 iterations within the significantly reduced computation time. In the future, the multiple modules will be built and a propagated error minimization method for multi-modules of ViSP will be studied.

\section{Acknowledgements}

This study was supported by the National Research Foundation of Korea (NRF) grant funded by the Korea government (No. 2009-0075397). It was also financially supported by Korea Minister of Ministry of Land, Transport and Maritime Affairs(MLTM) as 'U-City Master and Doctor Course Grant Program'.

\section{REFERENCES}

[1] Balageas D., Fritzen C. P. and Guemes A.(eds), Structural Health Monitoring, New Jersey: John Wiley \& Sons, Inc., 2006.

[2] Olaszek P., "Investigation of the dynamic characteristic of bridge structures using a computer vision method", Measurement 25, 227-236, 1999.

[3] Park J. W., Lee J. J., Jung H. J. and Myung H., "Vision-based displacement measurement method for high-rise building structures using partitioning approach", NDT \& E Int. 43, 642-647, 2010.

[4] Myung H., Lee S. M., and Lee B. J., "Paired structured light for structural health monitoring robot system", Struct. Health Monit. 10, 49-64, 2011.

[5] Jeon H., Bang Y., and Myung H., "A paired visual servoing system for 6-DOF displacement measurement of structures", Smart Mater. Struct. 20, 45019, 2011.

[6] Jeon H., Shin J. W., and Myung H., "Incremental Displacement Estimation of Structures using Paired Structured Light” Smart. Struct. Sys. 9, 273-286, 2012.

[7] Spencer B. F., Jr., Christenson R. E., and Dyke S. J., "Next generation benchmark control problem for seismically excited buildings", Proceedings of 2nd Int. Conf. on Structural Control, 1998. 